\title{
PUNCIÓN ASPIRACIÓN CON AGUJA FINA (PAAF) EN CLASIFICACIÓN DE NÓDULOS TIROIDEOS
}

\section{FINE NEEDLE ASPIRATION (FNA) TO CLASIFY THYROID NODULES}

\author{
Dr. Evelio Legal Balmaceda ${ }^{1}$, Dra.Cinthia Sosa Ramírez ${ }^{2}$
}

\section{RESUMEN}

OBJETIVO: Describir resultados de la PAAF en el diagnóstico etiológico de los nódulos tiroideos.

PACIENTES Y MÉTODOS: Estudio observacional, retrospectivo de pacientes con bocio que consultaron entre enero de 2006 y diciembre de 2013.

RESULTADOS: 42 pacientes estudiados, 33 (86\%) mujeres y 6 (14\%) hombres, entre 18 y 72 años (promedio43,65 años). Diagnóstico mediante PAAF: 8 (19\%) BETHESDA I, 24 (57\%) BETHESDA II, 9 (22\%) BETHESDA III, 1 (2\%) BETHESDA VI. Las categorías IV y V no se presentaron. Resultados anatomopatológicos: 22 (52\%) Bocio Coloide Multinodular, 5 (12\%) tiroiditis de Hashimoto, 4 (10\%) adenoma folicular, $4(10 \%)$ bocio coloide nodular, 4 (10\%) Carcinoma Papilar, $1(2 \%)$ carcinoma de células de Hurthle, 1 (2\%) Bocio multinodular quístico, 1 (2\%) carcinoma folicular. Las punciones BETHESDA I, 100\% (8 casos) fueron benignos. BETHESDA II, 95\% (23 DE 24)benignos, 5\% (1) correspondió a un carcinoma folicular. BETHESDA III, 55\% (5) benignos y $45 \%$ (4) malignos ( 3 carcinomas papilares y 1 carcinoma de células de Hurthle).BETHESDA VI, $100 \%$ ( 1 caso) carcinoma papilar.

CONCLUSIÓN: La PAAF es útil para estudiar neoplasias nodulares tiroideas. Presenta poca dificultad técnica, bajo costo y es mínimamente invasiva.

Palabras clave: PAAF, tiroides, Bethesda

\section{ABSTRACT}

OBJECTIVE: To describe FNA results in the etiological diagnosis of thyroid nodules.

PATIENTS AND METHODS: Observational, retrospective study of patients with goiter who consulted between January 2006 and December 2013.

RESULTS: 42 patients studied, 33 (86\%) women and $6(14 \%)$ men, between 18 and 72 years (average 43.65 years). Diagnosis by FNAP: 8 (19\%) BETHESDA I, 24 (57\%) BETHESDA II, 9 (22\%) BETHESDA III, $1(2 \%)$ BETHESDA VI. Categories IV and V were not submitted. Pathological results: 22 (52\%) Multinodular colloid goiter, 5 (12\%) Hashimoto's thyroiditis, $4(10 \%)$ follicular adenoma, $4(10 \%)$ nodular colloid goitre, 4 (10\%) Papillary carcinoma, 1 (2\%) ) Hurthle cell carcinoma, 1 (2\%) cystic multinodular goiter, 1 (2\%) follicular carcinoma. BETHESDA I punctures, 100\% (8 cases) were benign. BETHESDA II, 95\% (23 OF 24) benign, $5 \%$ (1) corresponded to a follicular carcinoma. BETHESDA III, $55 \%$ (5) benign and 45\% (4) malignant (3 papillary carcinomas and 1 Hurthle cell carcinoma). BETHESDA VI, 100\% (1 case) papillary carcinoma.

CONCLUSION: FNAB is useful to study nodular nodular neoplasms. It presents little technical difficulty, low cost and is minimally invasive
Keywords: FNAB, thyroid, Bethesda

\section{INTRODUCCIÓN $\mathbf{N}^{(1-9)}$}

La punción aspiración con aguja fina (PAAF) tiene un papel esencial en la evaluación de los pacientes eutiroideos con un nódulo tiroideo. Ella reduce la tasa de cirugía de tiroides innecesaria para los pacientes con nódulos benignos y, apropiadamente, clasifica pacientes con cáncer de tiroides para la cirugía.

Antes del uso rutinario de la PAAF de tiroides, el porcentaje de nódulos de tiroides resecados quirúrgicamente que eran malignos fue del $14 \%$. Con la práctica actual de la PAAF tiroidea, el porcentaje de nódulos resecados que son malignos supera el $50 \%$.

Es un procedimiento que ha ido paulatinamente introduciéndose en la práctica clínica y que, sin perder su vigencia, constituye actualmente el método de elección en la aproximación diagnóstica de la patología tiroidea.

Dada la importancia de la PAAF en la patología tiroidea, se hace necesario realizar un estudio sobre los resultados locales de la misma en nuestro servicio.

\section{OBJETIVOS}

General:

1. Describir los resultados de la punción aspiración con aguja fina realizada en el diagnóstico de la etiología de los nódulos tiroideos.

\section{Específicos:}

1. Describir la técnica de la punción aspiración con aguja fina que se practica en los nódulos tiroideos.

2. Describir las características demográficas de los pacientes con bocio en quienes se practicó PAAF.

3. Listar los diagnósticos pre operatorios de los pacientes con bocio y en los que se realizó PAAF.

4. Describir los resultados obtenidos en las PAAF, que fueron realizadas en esta casuística.

5. Relacionar las PAAF con los diagnósticos de anatomía patológica de los pacientes operados.

1. Especialista en cirugía general. Facultad de Ciencias Médicas, Universidad Nacional de Asunción. Paraguay

2. Médico Interno. Facultad de Ciencias Médicas, Universidad Nacional de Asunción. Paraguay

Autor correspondiente: Dr. Evelio Legal Balmaceda. Correo electrónico: eveliolegal@gmail.com

Artículo recibido: 7.09.17 - Artículo aceptado: 29.10.17

Autofinanciado por los autores. Los autores no declaran ningún conflicto de interés. 


\section{METODOLOGÍA}

\section{Diseño del estudio}

- Observacional, descriptivo, retrospectivo, de corte transversal.

Población

- Pacientes con bocio que consultaron en el Servicio de Cirugía de Sala X del Hospital de Clínicas de la FCM - U.N.A. de enero de 2006 a diciembre de 2013.

Criterios de inclusión

- Pacientes de ambos sexos, mayores de 18 años, con bocio, a quienes se les realizó PAAF.

- Historia clínica completa.

- Informe de PAAF completo.

- Informe de anatomía patológica completo.

Variables

- Edad.

- Sexo.

- Diagnóstico preoperatorio.

- Diagnóstico por PAAF.

- Diagnóstico anatomopatológico.

\section{Instrumentos}

Los datos fueron recogidos a través de cuestionarios completados mediante la revisión de las historias clínicas que contengan las variables de interés.

\section{Aspectos estadísticos}

Los datos recolectados fueron introducidos en una planilla electrónica Microsoft - Office Excell ${ }^{\circledR} 2010$ y posteriormente analizados empleando estadística descriptiva tales como porcentajes, distribución de frecuencias para variables categóricas, media, mediana, desvío standard y percentiles para las variables numéricas.

Para el cálculo del tamaño de la muestra de un estudio descriptivo de una variable dicotómica (presencia-ausencia de Ca. en la PAAF). Si se establece el valor de la proporción esperada $(P)$ en 0.20 , y la amplitud total del intervalo de confianza (W) en 0,30, se establece el tamaño de la muestra en 27 para un nivel de confianza de $95 \%$.

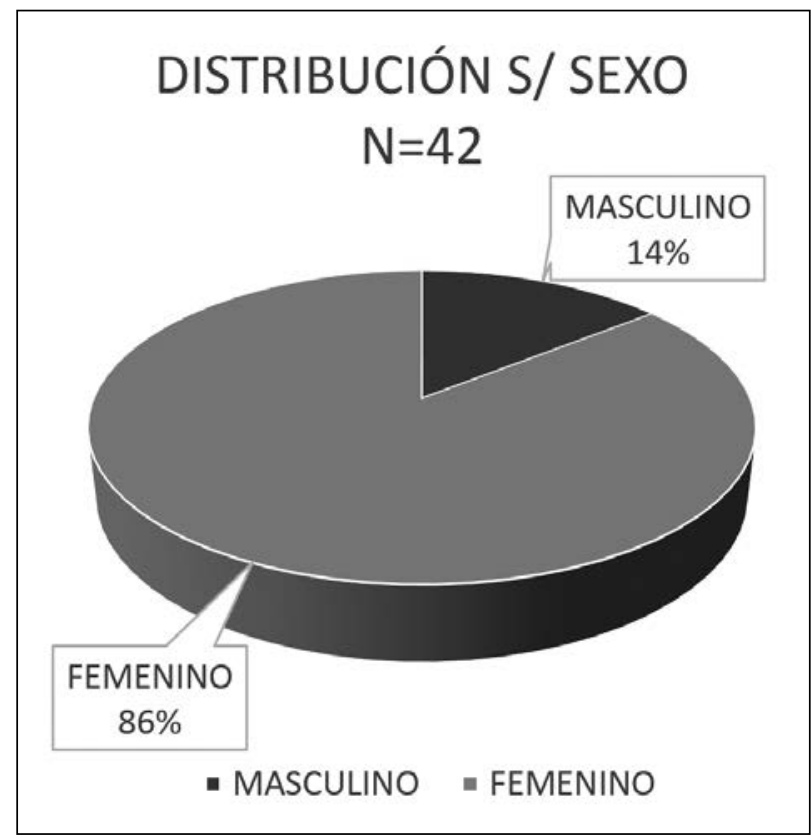

Gráfico 1.

\section{Aspectos éticos}

El estudio se llevó a cabo en pacientes ya tratados, con el consentimiento del Jefe de Cátedra del Servicio de Cirugía.

Los datos de las historias clínicas de los pacientes fueron manejados de modo confidencial.

\section{RESULTADOS}

- Se revisaron 122 fichas de pacientes operados (tiroidectomía) en el Servicio de Cirugía de Sala X entre enero de 2006 y diciembre de 2013.

- 42 fichas reunían todos los criterios de inclusión y las variables a estudiar.

- $33(86 \%)$ correspondían a pacientes de sexo femenino y 6 (14\%) a pacientes de sexo masculino. (Gráfico 1)

- Las edades comprendidas entre 18 y 72 años, promedio de edad de 43,65 años.

- El diagnóstico mediante PAAF fue: 8 (19\%) no diagnóstica (BETHESDA I), 24 (57\%) benigna (BETHESDA II), $9(22 \%)$ atipia de significado indeterminado (BETHESDA III), 1 paaf (2\%) fue maligna (BETHESDA VI). Las categorías IV y V no se presentaron. (Gráfico 2)

- Los resultados del examen anatomopatológico: 22 (52\%) de Bocio Coloide Multinodular (BCMN), 5 (12\%) de tiroiditis de Hashimoto, 4 (10\%) de adenoma folicular, 4 (10\%) de bocio coloide nodular, 4 (10\%) de Carcinoma Papilar, 1 (2\%) carcinoma de células de Hurthle, 1 (2\%) Bocio multinodular quístico, 1 (2\%) carcinoma folicular. (Gráfico 3)

- La distribución de los resultados de la PAAF preoperatoria en relación al resultado anatomopatológico posoperatorio (Gráfico 4) es como sigue:

- BETHESDA I (8 casos): 6 fueron BCMN, 1 adenoma folicular, 1 bocio multinodular quístico.

- BETHESDA II (24 casos): 14 BCMN, 4 tiroiditis de Hashimoto, 3 adenoma folicular, 2 bocio coloide nodular, 1 carcinoma folicular.

- BETHESDA III (9 casos): 2 BCMN, 1 tiroiditis de

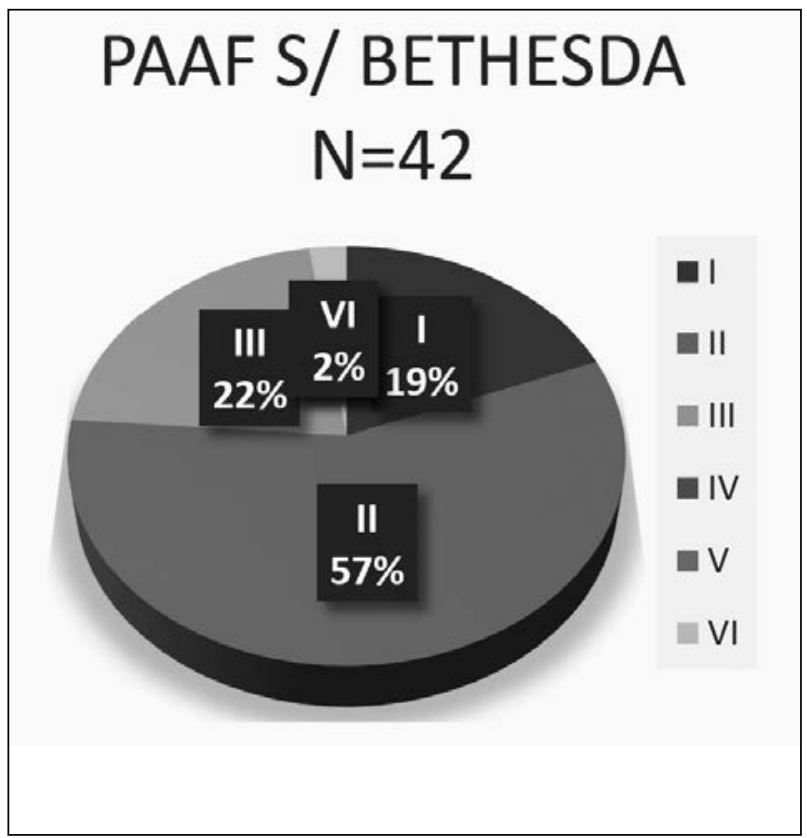

Gráfico 2. 


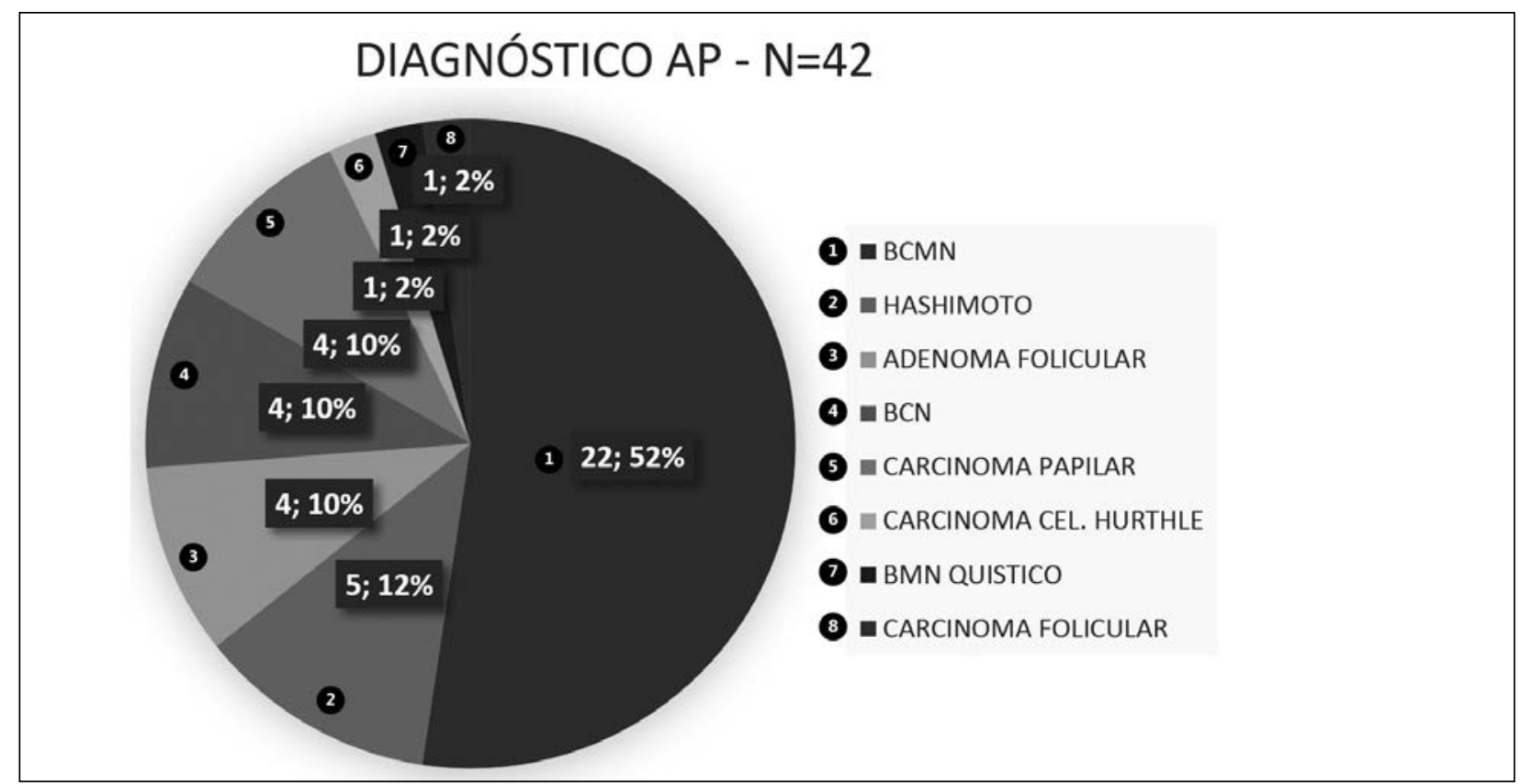

\section{Gráfico 3.}

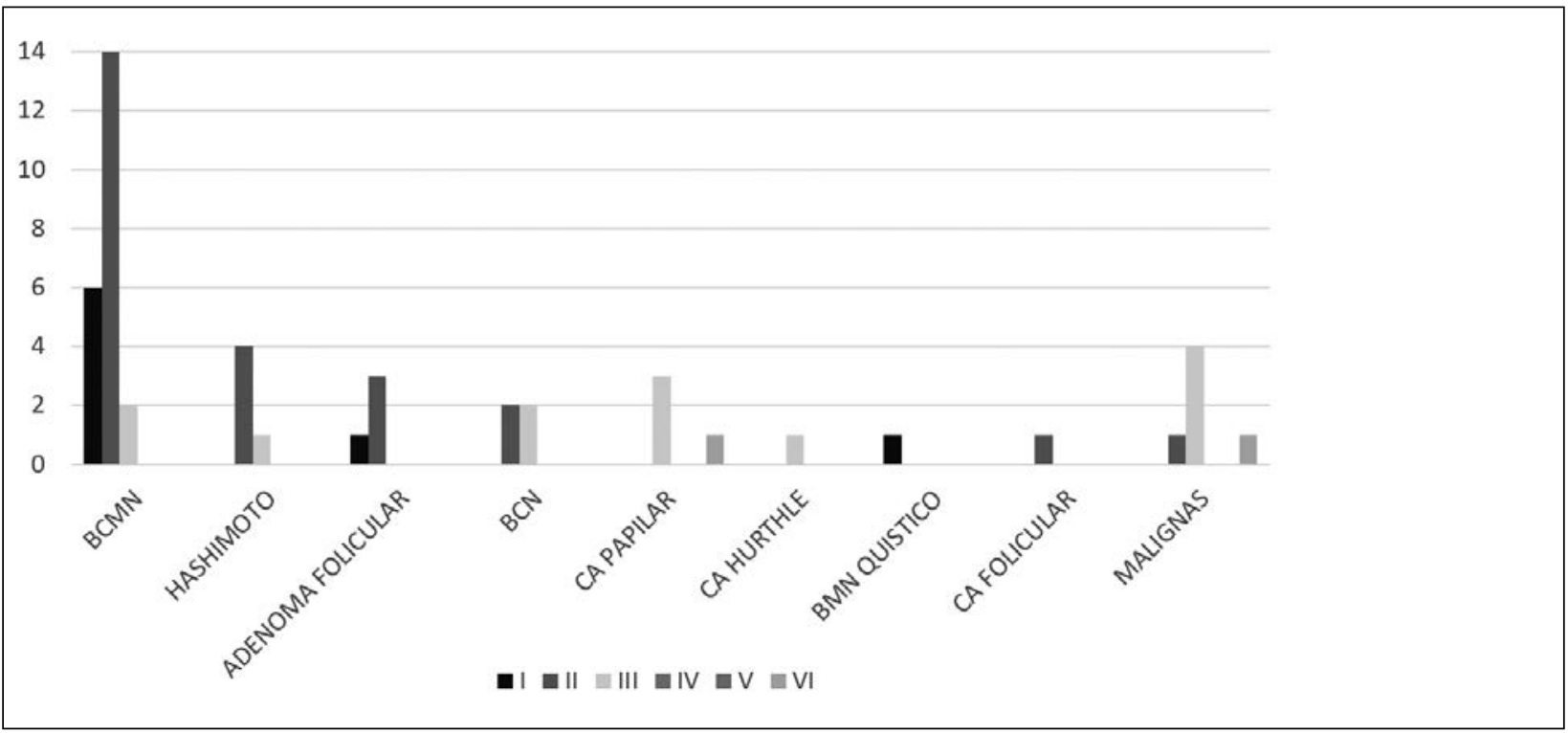

Gráfico 4.

\section{DIAGNÓSTICO PRE OPERATORIO}

NÓDULO TIROIDEO 2

$\mathrm{BN}=3$

CAPAPILAR $\square 1$

BMN 36

$\begin{array}{lllllllll}0 & 5 & 10 & 15 & 20 & 25 & 30 & 35 & 40\end{array}$ 
Hashimoto, 2 BCN, 3 carcinoma papilar, 1 carcinoma de células de Hurthle.

- BETHESDA VI (1 caso): 1 carcinoma papilar.

- Las categorías IV y V no se presentaron.

- En cuanto al diagnóstico preoperatorio, 36 casos correspondieron a bocio multinodular, 3 a bocio nodular, 2 a nódulo tiroideo y 1 caso a carcinoma papilar. (Gráfico 5)

- De las punciones NO DIAGNÓSTICAS (BETHESDA I), $100 \%$ (8 casos) correspondieron a hallazgos anatomopatológicos benignos.

- De las punciones BENIGNAS (BETHESDA I), 95\% (23 DE 24) coincidieron con resultados anatomopatológicos benignos, 5\% (1 caso) correspondió a un carcinoma folicular.

- De las punciones con ATIPIA DE SIGNIFICADO INDETERMINADO (BETHESDA III), 55\% (5 casos) correspondieron a casos benignos y $45 \%$ (4 casos) correspondieron a casos malignos (3 carcinomas papilares y 1 carcinoma de células de Hurthle).

- De las punciones MALIGNAS (BETHESDA VI), $100 \%$ (1 caso) resultó ser un carcinoma papilar.

\section{DISCUSIÓN}

La punción aspirativa con aguja fina (PAAF) de la tiroides es sugerida en la actualidad para la valoración inicial del nódulo tiroideo, así como de otras afecciones de la glándula tiroides, y se señala en estudios realizados en adultos que posee gran sensibilidad de hasta $100 \%$ (capacidad de detectar lesiones malignas) y una gran especificidad de aproximadamente $47 \%$ (capacidad de diferenciar lesiones benignas de malignas) ${ }^{(10-14)}$.

La distribución de la patología tiroidea estudiada en este trabajo, coincide en distintos estudios en relación a la edad ${ }^{(10-}$ ${ }^{14)} \mathrm{y}$ al sexo, donde existe un claro predominio del sexo femenino $^{(15-18)}$.

En la mayoría de los trabajos predomina el resultado BENIGNO de la PAAF, con porcentajes similares al de este estudio $^{(10-17)}$.

Con relación al resultado anatomopatológico, en la mayoría de los trabajos predominó entre los resultados benignos diagnóstico de ADENOMA FOLICULAR ${ }^{(15,17,18)}$ a diferencia del encontrado en este trabajo que correspondió a BOCIO MULTINODULAR $^{(16)}$.
BIBLIOGRAFIA

1. Cooper DS, Doherty GM, Haugen BR, Kloos RT, Lee SL, Mandel SJ, et al: Revised American Thyroid Association management guidelines for patients with thyroid nodules and diiferentiated thyroid cáncer. Thyroid 2009 19:1167-214.

2. Cibas ES, Ali SZ. The Bethesda System for Reporting Thyroid Cytopathology. Thyroid. 2009 Nov;19(11):1159-65.

3. Pacini F, Castagna MG, Brilli L, Pentheroudakis G. Thyroid cáncer: ESMO clinical practice guidelines for diagnosis, treatment and follow-up. Annals of Oncology23 2012 (Supplement 7):110-19.

4. Arrechedera C, Oviedo N, Linares Y, Sanabria E, Canozo L. La punción con aguja fina método de estudio diagnóstico en nódulos de la glándula tiroides. Rev. venez. oncol. 2009 Jun; 21(2): 77-84.

5. Gharib H, Goellner JR. Fine-needle aspiration biopsy of the thyroid: an appraisal. Ann Intern Med. 1993 15;118(4):282-9.

6. Gharib H, Goellner JR, Johnson DA. Fine-needle aspiration cytology of the thyroid. A 12-year experience with 11,000 biopsies. Clin Lab Med. 1993 Sep;13(3):699-709.

7. Holzheimer RG. Benign nodular thyroid disease. In: Holzheimer RG, Mannick JA, editors. Surgical Treatment: Evidence-Based and Problem-Oriented. Munich: Zuckschwerdt; 2001.

8. Sierra Fonseca RM, Escalona Veloz R, Galano Stivens E, Cogle Duvergel YY, Medina Tola J.. Comparación entre los resultados citológicos e histológicos de pacientes con afecciones tiroideas. MEDISAN. 2014 Jun;18(6): 833-40.

9. Granados-García M, Cortés-Flores AO, González-Ramírez IC, Cano-Valdez AM, Flores-Hernández L, Aguilar-Ponce JL. Neoplasia folicular de la tiroides: la importancia de la correlación clínica y citológica. Cir Cir. 2010; 78 (6): 476-81.

10. Piñeiro Lamas R, Torres Gómez de Cádiz A, Miranda Espinosa N, Cárdenas Bruno M, García Fors R. Biopsia aspirativa con aguja fina del tiroides. Rev Cubana Pediatr 1996 Ago;68(2): 119-24.

11. Blum M. The diagnosis of thyroid nodule using aspiration biopsy and cytology. Arch Intern Med 1984; 144:1140.

12. Mahomey P. Diferential diagnosis of goiter. Ped Clin North Am 1987;34(4):891.

13. Arnold AA, Georgitis N, Waldrow E, Sims, J Kidd G. Fine needle aspiration of the thyroid use in an average health care facility. Am J Med 1987;83:489.

14. Aschcraft MW, Van Herle AJ. Management of thyroid nodules. History and physical examination, blood test and ultrasonography. Head Neck Surg 1981;3:216

15. Ochoa Torres F, Gil León R. Biopsia por punción con aguja fina sin aspiración en el diagnóstico prequirúrgico del nódulo del tiroides. Rev Cubana Endocrinol. 2001 Dic;12(3):139-44.

16. Chala A, Pava R, Franco H, Álvarez A, Franco A. Criterios ecográficos diagnósticos de neoplasia maligna en el nódulo tiroideo: correlación con la punción por aspiración con aguja fina y la anatomía patológica. rev. colomb. cir. 2013 Mar; 28(1): 15-23.

17. Orué J, Saavedra J. Patología quirúrgica de la glándula tiroides. An. Fac. med. 2008, vol.69, n.3, pp. 182-87.

18. Legra E, Corría Y, Montero Y. Caracterización clínico quirúrgica y anatomopatológica de pacientes con cáncer de tiroides en el período 1995-2011. MEDISAN 2013 Nov;17(11): 8064-9. 